\title{
Perinatal Cat and Dog Exposure and the Risk of Asthma and Allergy in the Urban Environment: A Systematic Review of Longitudinal Studies
}

\author{
Caroline J. Lodge, ${ }^{1,2}$ Katrina J. Allen, ${ }^{2,3}$ Adrian J. Lowe, ${ }^{1,2}$ David J. Hill, ${ }^{2}$ Cliff S. Hosking, ${ }^{4}$ \\ Michael J. Abramson, ${ }^{5}$ and Shyamali C. Dharmage ${ }^{1,2}$ \\ ${ }^{1}$ Centre for Molecular, Environmental, Genetic and Analytic Epidemiology, School of Population Health, University of Melbourne, \\ Melbourne, Vic 3010, Australia \\ ${ }^{2}$ Murdoch Children's Research Institute, Royal Children's Hospital, Melbourne, Vic 3052, Australia \\ ${ }^{3}$ Department of Allergy, and Department of Paediatrics, University of Melbourne, Royal Children's Hospital, Melbourne, \\ Vic 3052, Australia \\ ${ }^{4}$ John Hunter Children's Hospital, Newcastle, NSW 2305, Australia \\ ${ }^{5}$ Department of Epidemiology and Preventive Medicine, Monash University, The Alfred Hospital, Melbourne, Vic 3004, Australia
}

Correspondence should be addressed to Caroline J. Lodge, clodge@unimelb.edu.au

Received 2 July 2011; Accepted 26 August 2011

Academic Editor: Shau-Ku Huang

Copyright (c) 2012 Caroline J. Lodge et al. This is an open access article distributed under the Creative Commons Attribution License, which permits unrestricted use, distribution, and reproduction in any medium, provided the original work is properly cited.

Background. The literature is contradictory concerning pet exposure and the risk of development of asthma and other allergic diseases. Using longitudinal studies, we aimed to systematically review the impact of pet ownership in the critical perinatal period as a risk factor for allergies in childhood. Methods. Medline database was searched for urban cohort studies with perinatal exposure to cats and/or dogs and subsequent asthma or allergic disease. Results. Nine articles, comprising 6498 participants, met inclusion criteria. Six found a reduction in allergic disease associated with perinatal exposure to dogs or, cats or dogs. One study found no association. Two found increased risk only in high-risk groups. Conclusion. Longitudinal studies in urban populations suggest that perinatal pets, especially dogs, may reduce the development of allergic disease in those without a family history of allergy. Other unmeasured factors such as pet-keeping choices in allergic families may be confounding the association seen in these high-risk families, and further study is required.

\section{Background}

Allergic disease appears to be on the rise worldwide, and although an allergic family history is one of the strongest risk factors for childhood allergy [1], large international studies [2-4] which highlight geographical differences in allergy prevalence, strongly suggest that environmental influences also play a causal role. Although pets are known to aggravate asthma, allergic rhinitis, and eczema in sensitized individuals [5] controversy remains about whether early life pet exposure is a risk factor or a protective factor in their development. Current guidelines issued in Australia [6], the United States [7], and the United Kingdom [8], and by the Global Initiative for Asthma [9] all agree there is currently insufficient evidence to provide any recommendations in relation to petkeeping in early life and the development of asthma and allergic disease because systematic reviews [10-13] and a meta-analysis [14] have reached different conclusions. Early reviews [10] found pet-keeping increased the risk of sensitization [10] and allergic disease $[10,12]$ with later reviews $[11,13]$ finding no effect. A recent meta-analysis [14] reported less risk of childhood asthma associated with cats, but increased risk with dogs.

These disparate findings may be partly explained by inclusion of articles with different study designs. To date, there are no randomized controlled trials (RCTs) on the effect of 
pet exposure on allergic disease outcomes. In the absence of RCTs, the most valuable evidence is provided by longitudinal studies with a wealth of baseline data and frequent followups which enable assessment of pet exposure prior to the outcome of allergic disease. Despite this, all the current reviews have included at least two study designs (case control and cohort) [14] with the remainder also including crosssectional studies [10-12].

Differences in the timing of exposures between studies may also provide a reason for varied results. It has been proposed that there are important windows of immune development [15] in which environmental exposures can either increase or decrease the risk of subsequent allergic disease development [16]. The perinatal period encompassing 20 weeks prior to birth until 4 weeks after is a critical time in developmental maturation of the immune system [17]. There is good evidence that the developing immune system in the fetus is susceptible to environmental influences and that immune development in utero is epigenetically regulated [17] with maternal exposures influencing the child's propensity for allergic disease $[18,19]$. To date no reviews have limited assessment of pet keeping exposure at the critical perinatal period which may have a differential effect on risk than pet exposure at other periods of life. Lastly, another source of difference between studies is the varied study settings especially urban versus rural environment. A key relevant difference is the way in which pets are kept and the interaction between pets and other animals in rural settings. Hence, the clearest way to tease out the effects of cat and dog exposure on asthma and allergy in children would be to study this in an urban environment. None of the reviews have taken this into account.

Therefore, we have conducted a systematic review of longitudinal studies in urban environments to explore the relationships between cat and dog exposure in the perinatal period and subsequent asthma or allergy.

\section{Methods}

\subsection{Inclusion Criteria}

(i) Human.

(ii) Full-term infants.

(iii) Population based or allergy enriched sample.

(iv) Exposure to cat and/or dog presence or allergen levels measured and reported from 20 weeks prior to birth until 4 weeks after birth.

(v) Urban households only.

(vi) Outcome assessed and reported-any allergic disease (asthma/wheeze/eczema/allergic rhinitis/food allergy) or atopy/sensitization as measured by serum IgE (total or specific) or on Skin Prick Testing.

(vii) Longitudinal (cohort) studies.

The comparison groups were the children not exposed to pets within each study.

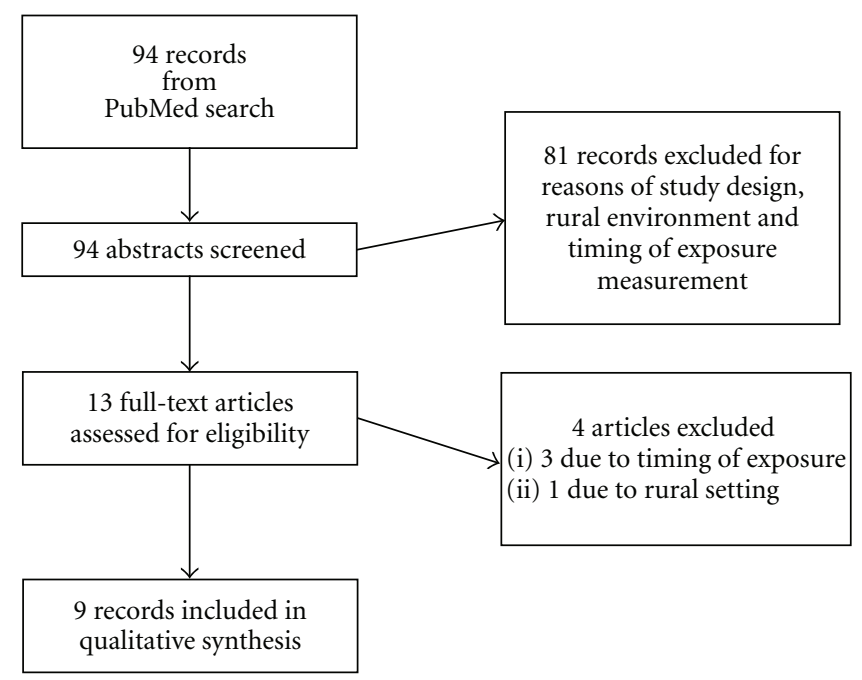

FIGURE 1: Flow chart of searching process.

2.2. Search Strategy. We searched Medline using the following strategy in PubMed. The last search date was 17 May 2011.

(i) One or more allergic disease outcome term: Allergy and Immunology "[Mesh] OR "Hypersensitivity" [Mesh]) OR "Asthma" [Mesh] OR "Respiratory Sounds" [Mesh] OR "Rhinitis" [Mesh] OR "Eczema" [Mesh] OR "Dermatitis, Atopic" [Mesh] OR “Immunoglobulin E" [Mesh]) OR "Bronchial Hyperreactivity" [Mesh]) OR "Food Hypersensitivity” [Mesh] OR “Allergens” [Majr]

AND

(ii) One or more pet exposure term: "Pets" [Mesh] OR "Animals, Domestic" [Mesh] OR "Cats" [Mesh], OR "Dogs" [Mesh]

AND

(iii) One or more age of exposure term: "Prenatal Exposure Delayed Effects" [Mesh] OR "Maternal exposure" [Mesh], OR "Fetus" [Mesh], OR "Infant, Newborn" [Mesh] OR "Birth"

AND

(iv) Study type-cohort, NOT review.

2.3. Process for Selecting Studies. A flow chart of the study selection process is shown in Figure 1. One author assessed all abstracts for eligibility. Full-text articles of eligible abstracts were then further assessed by the same author.

\section{Results}

Information concerning population, study type, exposure, outcome, and consideration of interaction by familial allergy for each of the nine studies is presented in Table 1. 


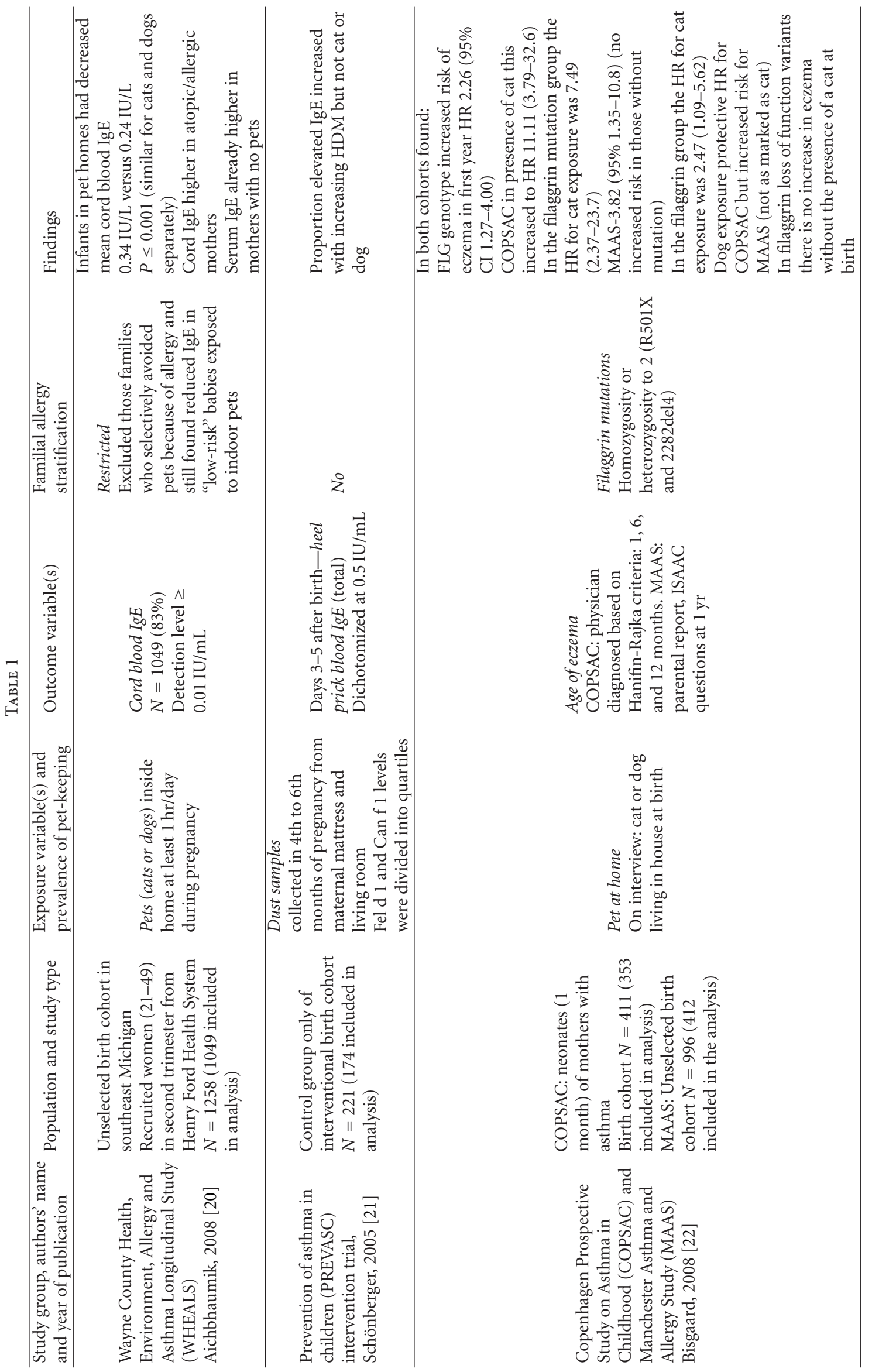




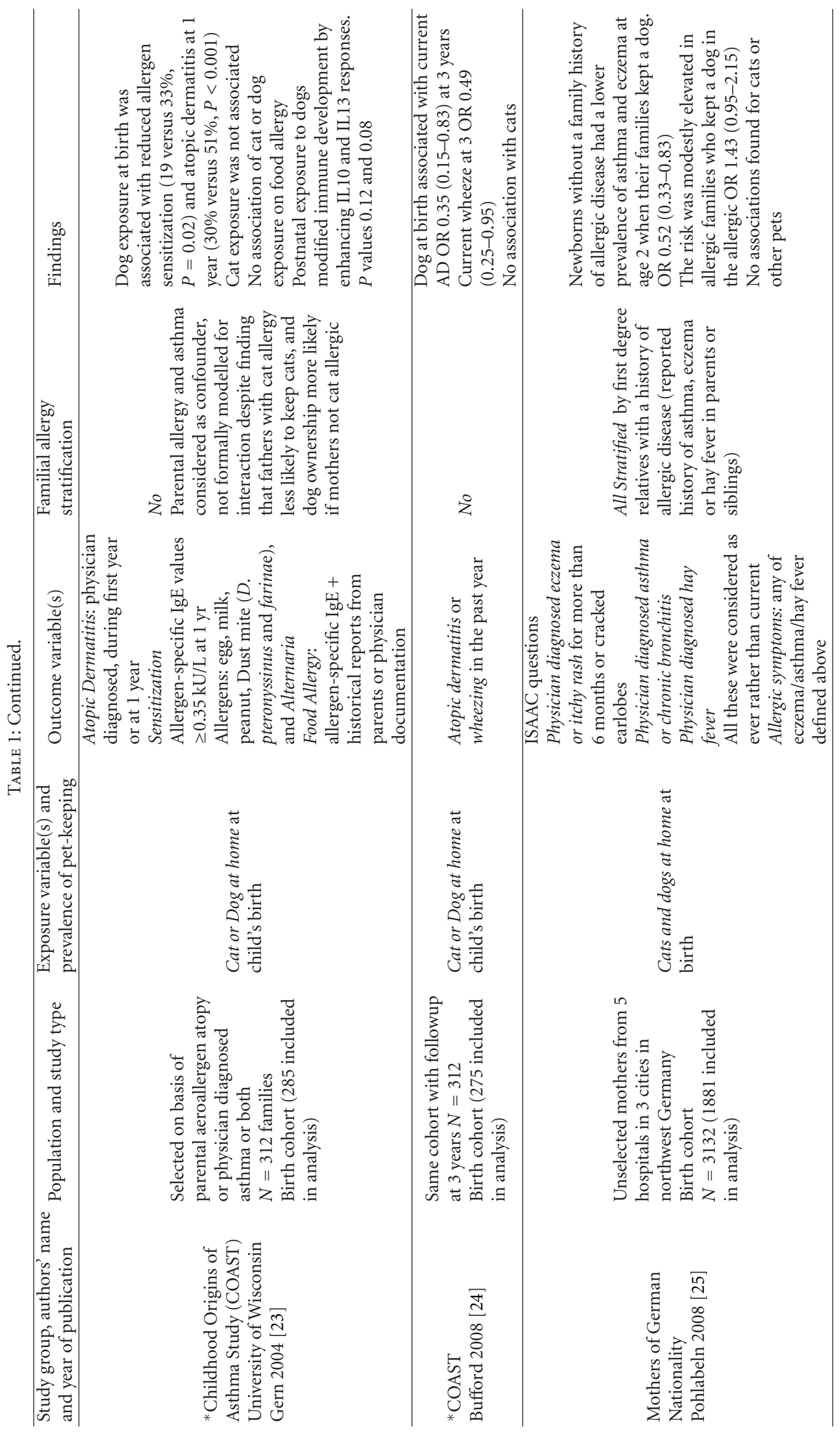




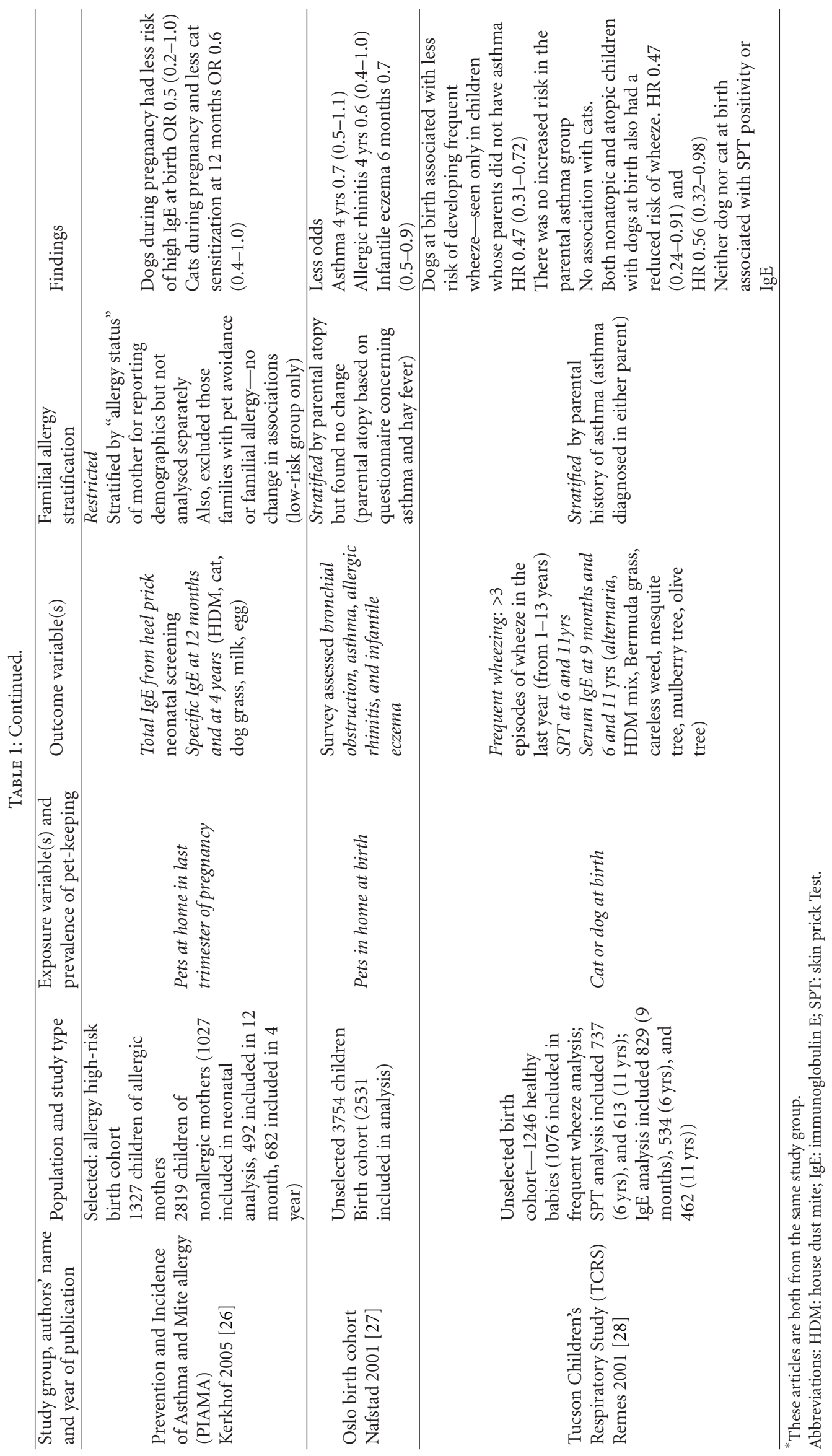


The nine included articles represented 9 different studies. There were two articles included from one study, with outcomes presented at both 1 year [23] and 3 years [24] of age. Additionally one article reported on the findings of two studies [22]. Six studies were population based and three were on children at increased risk of allergic disease. The numbers analyzed ranged from 174 [26] to 2531 [27], while the total population included across all studies was 6,498 . Only one article reported pet exposure exclusively as quartiles of allergen levels in vacuumed dust [21]; all other articles simply recorded the presence of cats and/or dogs in the home.

The allergic outcomes reported included: eczema in 4 articles; asthma/wheeze in 3; neonatal IgE in 3; sensitization in 2; allergic rhinitis in 1; food allergy in 1, and allergic symptoms (combination of eczema, asthma and hay fever) in one. The definitions and ages at which these outcomes were assessed varied between studies.

When assessing the quality of observational studies, particularly for possible sources of bias, the importance of addressing the following essential areas has been highlighted [29]: appropriate selection of participants, appropriate measurement of variables, and appropriate control of confounding. An assessment of the quality of included studies with reference to these areas is presented in Table 2.

Selection of participants and measurements of exposure and outcome variables were not thought to be sources of bias in any of the studies. None of the studies commented on recall bias although most of them recorded a parental history of allergy (a possible source of bias) retrospectively. Most of the studies used questionnaires for collecting data, so that interviewer bias was not applicable. Where interviews were performed however, whether or not the interviewer was blinded to the pet exposure status was not mentioned. There was possible bias due to loss to followup in four articles which did not explore whether those missing were different from those remaining in the study [22, 24, 26, 27]. Included confounders varied between studies introducing a possible source of bias.

The studies were divided into two groups based on when pet exposure was recorded. There were three studies which reported prenatal cat and dog exposure, and nine which reported exposure to cat and dog in the neonatal period.

\subsection{Exposure Recorded Prenatally [20, 21, 26]}

3.1.1. Neonatal IgE. The main outcome from all three articles was the total level of neonatal IgE, measured from cord blood [20] or from the neonatal screening heel prick test at 3-5 days after birth $[21,26]$. A lower level of IgE at birth was found in two articles $[20,26]$ if dogs [26], or cats or dogs [20] were kept during pregnancy. Both of these articles further restricted their analysis to a subgroup of low-allergy-risk infants and confirmed a lower level of neonatal IgE. The third article, which found no association [21] between neonatal IgE and pet exposure prenatally, was not strictly comparable having used levels of cat and dog allergens in dust as the measures of pet exposure.
3.1.2. Sensitization. Additionally one of the articles [26], which was based on a selected allergy risk population, found a lower risk of IgE sensitization to cat at 12 months in those children whose mothers had been exposed to cats during pregnancy [26].

3.2. Exposure Recorded Postnatally [22-25, 27, 28]. There were six articles which recorded postnatal allergen exposure [22-25, 27, 28]. Although these articles recorded pet or pet allergen exposure in the first 4 weeks of life, almost all mothers would certainly have also had exposure during pregnancy.

3.2.1. Eczema. Four articles reported eczema as an outcome, variably defined as infantile eczema [27], atopic dermatitis $[23,24]$, or physician diagnosed eczema [22] and assessed at 6 months [27], 1 year [22, 23], or 3 years [24] of age.

In three of these articles $[23,24,27]$ (representing two studies), the risk of atopic dermatitis or infantile eczema was reduced for children exposed to dogs [23, 24] or pets [27] at birth. The Childhood Origins of Asthma Study (COAST) $[23,24]$ followed up a high-risk birth cohort and found a lower risk of atopic dermatitis by 1 year and at 3 years when exposed to dogs at birth. There was no association with cats. In an unselected population from Oslo [27], it was reported that pets at birth conferred less risk of infantile eczema by 6 months of age. When stratified by paternal atopy (asthma and hay fever) only the high-risk group still showed less atopic eczema risk.

In the other article [22] (representing two studies), the exposure groups were stratified by genetic/hereditary factors and the risk of eczema was increased only in the high-risk groups if exposed to a pet. Bisgaard et al. [22] identified children with either of 2 filaggrin-(FLG)-null mutations in both the Copenhagen Prospective Study on Asthma in childhood (COPSAC) and the Manchester Asthma and Allergy study (MAAS) birth cohorts. When exposed to cats at birth, children with a FLG null mutation had an increased risk of eczema in the first year: there was no convincing evidence for a similar relationship with dog exposure at birth.

3.2.2. Wheeze/Asthma. Three articles measured asthma or wheeze as an outcome $[24,27,28]$. The variables measured included current wheeze at age 3 years [24], bronchial obstruction or asthma at age 4 years [27], and frequent wheezing ( $>3$ episodes per year) at ages 1-13 years [28]. All of these studies reported reduced odds or a reduced hazard ratio associated with a dog [24, 28] or with pets at birth [27]. No studies showed an association with cats alone.

Two of these three studies stratified by family history of allergy or parental asthma [27, 28], one [28] finding a risk reduction in the group without a parental history of asthma whilst the other found no change in risk [27].

3.2.3. Other Outcomes (Sensitization, Rhinitis, Food Allergy, or Combined Variable). There were three studies which 
TABLE 2: Evaluating the role of bias in the included studies.

\begin{tabular}{|c|c|c|c|c|c|c|c|c|c|}
\hline Study & $\begin{array}{c}\text { Aichbhaumik } \\
\text { [20] }\end{array}$ & $\begin{array}{c}\text { Schönberger } \\
{[21]}\end{array}$ & $\begin{array}{l}\text { Bisgaard } \\
{[22]}\end{array}$ & Gern $[23]$ & Bufford [24] & $\begin{array}{c}\text { Pohlabeln } \\
\text { [25] }\end{array}$ & $\begin{array}{l}\text { Kerkhof } \\
{[26]}\end{array}$ & $\begin{array}{c}\text { Nafstad } \\
{[27]}\end{array}$ & $\begin{array}{c}\text { Remes } \\
{[28]}\end{array}$ \\
\hline \multicolumn{10}{|c|}{ Methods for selecting study participants } \\
\hline $\begin{array}{l}\text { Appropriate source } \\
\text { population }\end{array}$ & ;) & :) & (:) & (;) & (:) & ;) & ;) & ;) & ;) \\
\hline $\begin{array}{l}\text { Inclusion and } \\
\text { exclusion criteria }\end{array}$ & ;) & (:) & ;) & (:) & ;) & ;) & ;) & ;) & ;) \\
\hline \multicolumn{10}{|c|}{ Methods for measuring exposure and outcome variables } \\
\hline $\begin{array}{l}\text { Appropriate } \\
\text { exposure } \\
\text { measurement }\end{array}$ & (:) & ;) & ;) & ;) & ;) & ;) & ;) & ;) & ;) \\
\hline $\begin{array}{l}\text { Appropriate } \\
\text { outcome } \\
\text { measurement }\end{array}$ & ;) & (:) & ;) & (:) & ;) & (;) & (:) & (:) & (:) \\
\hline \multicolumn{10}{|c|}{ Methods to deal with design-specific sources of bias } \\
\hline Recall bias & $\mathrm{NI}$ & NI & NA & $\mathrm{NI}$ & NI & $x$ & NI & NI & NI \\
\hline Interviewer bias & NI & NI & NI & NI & NI & NA & NA & NA & NA \\
\hline Loss to followup & (:) & (:) & $(1) \checkmark \times$ & (:) & $\times$ & NA & $\checkmark \times$ & $\checkmark \times$ & (:) \\
\hline Blinding & NA & NA & $\mathrm{NI}$ & NI & NI & NA & NA & NA & NA \\
\hline \multicolumn{10}{|c|}{ Methods to control confounding } \\
\hline Appropriate design & ;) & $\checkmark \times$ & $x$ & $\checkmark \times$ & $\checkmark \times$ & $\checkmark \times$ & $\checkmark \times$ & (:) & $\checkmark x$ \\
\hline $\begin{array}{l}\text { Appropriate } \\
\text { analytical methods }\end{array}$ & $\checkmark \times$ & (:) & $\times$ & ;) & (:) & ;) & $\checkmark \times$ & $\checkmark \times$ & ;) \\
\hline \multicolumn{10}{|c|}{ Statistical methods (excluding confounding) } \\
\hline $\begin{array}{l}\text { Appropriate } \\
\text { statistics }\end{array}$ & $\checkmark \times$ & $\checkmark \times$ & ;) & ;) & $\checkmark \times$ & ;) & $\checkmark \times$ & (:) & ;) \\
\hline \multicolumn{10}{|c|}{ Conflict of interests } \\
\hline $\begin{array}{l}\text { Declarations of } \\
\text { conflict of interests }\end{array}$ & (:) & $\times$ & ;: & $\times$ & $\times$ & $\times$ & $\times$ & $\times$ & $\times$ \\
\hline $\begin{array}{l}\text { Identification of } \\
\text { funding sources }\end{array}$ & ;) & (:) & ; & ;) & ; & ;) & (:) & (:) & (:) \\
\hline \multicolumn{10}{|c|}{ NHMRC evidence grading (ref) } \\
\hline Levels & III-2 & III-2 & III-2 & III-2 & III-2 & III-2 & III-2 & III-2 & III-2 \\
\hline
\end{tabular}

NA: not applicable; NI: not described in article.

(): adequate; $\checkmark \times$ : poor; $\times$ : not done.

measured sensitization as an outcome. Two of these showed no association $[28,30]$ with pet exposure at birth, whilst the third reported reduced allergen sensitization at 1 year in those children exposed to a dog at birth [23]. One article [27] examined allergic rhinitis at age 4 years and found a reduced risk in children exposed to pets at birth which persisted in both groups following stratification for parental atopy.

Only one article reported on the outcome of food allergy. This study found no effect of cat or dog at birth on the risk of "confirmed food allergy" [23] (defined as specific IgE to egg milk or peanut of $\geq 0.35 \mathrm{kU} / \mathrm{L}$ and a convincing history).

One article [25] reported "allergic symptoms" at 2 years of age (physician diagnosed eczema, chronic bronchitis or asthma, or hay fever) and found a reduction in symptoms among the children of families without a history of allergic disease if they had kept dogs at birth. However in dogkeeping families with a history of allergic disease, a modest increase was found.
3.2.4. Summary of Results. Of the nine studies which were included, six [20, 23, 24, 26-28] found that the risk of allergic disease, or allergic sensitisation, was reduced in children who had been exposed to pets at or before birth. Dogs were associated with less risk of allergic outcomes in four $[23,24,26,28]$ of the six articles, while the remaining two found either pet to be associated with a reduced risk [20,27]. Additionally one article reported a lower risk associated with cat exposure [26]. Only one small study $(n=174)$ failed to find an association [21].

Measures of familial allergy or pet avoidance were employed by four $[20,26-28]$ articles to account for the confounding aspects of pet-keeping choices. Limiting the analysis to the non-allergy-prone low-risk group did not change the associations in two articles $[20,26]$. The remaining two articles $[27,28]$ stratified their analyses by familial high- and low-risk allergy groups. Subsequently, Nafstad et al. [27] found reduced odds of allergic disease 
in both high- and low-risk groups, whilst Remes et al. [28] found the reduced risk of allergic disease in pet-exposed children was limited to the low-risk familial allergy group.

This finding was further supported by the remaining two articles $[22,25]$ which only presented results stratified by allergic predisposition. Both found that high-risk children had an increased risk of allergic outcomes in the presence of dog or cat exposure at birth.

\section{Discussion}

Overall we found that for children without a family history of allergy, owning a dog was protective against the development of allergic disease. By contrast the findings with regard to those with a family history of allergy were more difficult to interpret. A major confounding factor may be that petkeeping behaviour is likely to be strongly influenced by the allergic status of the parents and siblings, as is the child's risk of allergic disease.

Further work assessing the impact of allergic status on owning a pet will be required to better understand whether this effect is due to a gene-environment interaction.

One of the problems in this field of research as outlined above is the inability to completely account for the confounding effects of pet-keeping choices made by allergic families. Families with allergic members are less likely to keep pets [31,32], so it may appear that allergic disease is associated with not keeping pets. The lack of randomized controlled trials of pet-keeping, which would remove the confounding effect associated with pet-keeping choices in allergic families, makes the next best evidential study design a prospective birth cohort [33]. The ability of cohort investigators to manage the effects of confounding by familial allergy depends upon the validity and completeness of the information they have gathered concerning familial allergy and pet-keeping choices.

In this systematic review, we found that the treatment of confounding by familial allergy status varied greatly between articles (Table 2). Some of the articles limited their analyses to low-risk groups, which gave only the less interesting half of the picture. There was no uniform measure of familial predisposition, with some articles using only parental asthma, some using all first degree familial allergic disease or atopy and one study combining this with pet avoidance behaviour. The varied nature of the measurement of familial allergic predisposition made it less likely that a clear picture of the relationship between pet-keeping and allergic disease would emerge. The articles also differed in which factors they included in their analytical models as potential confounders.

Another problem with pooling studies for a systematic review is that they may measure outcomes at different ages. As part of a comprehensive systematic review, Chen et al. [11] grouped 21 birth cohort studies whose measured outcome of wheeze varied from 1 year [34] to 12 years [28]. The nature of wheeze at these two ages is very different. Over half of wheeze recorded in early childhood is transient [3537]. Therefore, studies reporting wheeze/asthma outcomes in early childhood will identify many children who will not go on to have true asthma at school age and may be less specific in their findings than those which measure wheeze after six years of age. This was also an issue for our articles where three included articles measured wheeze at or before four years of age.

Other issues related to the studies in this systematic review were the possibility of attrition bias due to loss of followup was not always explored, recall bias for survey questions related to parental allergies was not mentioned by any of the articles, and, reporting bias might also have influenced which articles were identified. Also, due to the perinatal exposure criterion, other birth cohort studies including the Multicentre Allergy Study [38], which measured pet exposure at 6 months, but not during the first month of life, were excluded.

Compared to previous reviews which have yielded inconsistent results, the strengths of this review are that it has measured pet exposure at one time period, the perinatal period which is arguably a critical exposure window for immune system maturation; it has included only one study design, the cohort study which is the design with the most evidential weight in this field; it has included only urban populations, thus avoiding other potentially confusing exposures present in rural communities; it has also identified the important role of familial allergy in interpretation of any results.

In the current systematic review, we were unable to perform a meta-analysis on the included articles due to heterogeneity in timing and assessment of exposures and outcomes. Despite this, the findings appear to have similarities across articles.

\section{Conclusion}

This paper of longitudinal studies of perinatal cat and/or dog exposure in urban populations suggests that dog exposure may have a protective effect on the risk of allergic disease in low-risk populations. Unfortunately in children at highrisk of allergic disease, there is still no clear answer. Further longitudinal studies or randomised controlled trials, in which the effect of familial allergy on pet-keeping choices is clearly explored, are needed.

\section{Acknowledgments}

C. J. Lodge is supported by The Sidney Myer Health Fund and the National Health and Medical Research Council of Australia (NHMRC); K. J. Allen, A. J. Lowe, and S. C. Dharmage are all supported by NHMRC.

\section{References}

[1] L. Lowe, A. Custovic, and A. Woodcock, "Childhood asthma," Current Allergy and Asthma Reports, vol. 4, no. 2, pp. 159-165, 2004.

[2] M. I. Asher, S. Montefort, B. Björkstén et al., "Worldwide time trends in the prevalence of symptoms of asthma, allergic rhinoconjunctivitis, and eczema in childhood: ISAAC Phases One and Three repeat multicountry cross-sectional surveys," The Lancet, vol. 368, no. 9537, pp. 733-743, 2006. 
[3] C. Janson, J. Anto, P. Burney et al., "The European community respiratory health survey: what are the main results so far?" European Respiratory Journal, vol. 18, no. 3, pp. 598-611, 2001.

[4] M. Masoli, D. Fabian, S. Holt, and R. Beasley, "The global burden of asthma: executive summary of the GINA Dissemination Committee Report," Allergy, vol. 59, no. 5, pp. 469-478, 2004.

[5] E. C. TePas, A. A. Litonjua, J. C. Celedón, D. Sredl, and D. R. Gold, "Sensitization to aeroallergens and airway hyperresponsiveness at 7 years of age," Chest, vol. 129, no. 6, pp. 1500-1508, 2006.

[6] S. L. Prescott and M. L. K. Tang, "The Australasian Society of Clinical Immunology and Allergy position statement: summary of allergy prevention in children," Medical Journal of Australia, vol. 182, no. 9, pp. 464-467, 2005.

[7] National Asthma Education and Prevention Program, "Expert Panel Report 3 (EPR-3): guidelines for the diagnosis and management of asthma-summary report 2007," Journal of Allergy and Clinical Immunology, vol. 120, no. 5, supplement, pp. S94-S138, 2007.

[8] G. Douglas, B. Higgins, N. Barnes et al., "British guideline on the management of asthma: a national clinical guideline," Thorax, vol. 63, no. 4, pp. iv1-iv121, 2008.

[9] E. D. Bateman, S. S. Hurd, P. J. Barnes et al., "Global strategy for asthma management and prevention: GINA executive summary," European Respiratory Journal, vol. 31, no. 1, pp. 143-178, 2008.

[10] A. Ahlbom, A. Backman, J. Bakke, T. Foucard, S. Halken, and N.-I. M Kjellman, "NORDPET: pets indoors: a risk factor for or protection against sensitization/ allerg," Indoor Air, vol. 8, pp. 219-235, 1998.

[11] C. M. Chen, C. Tischer, M. Schnappinger, and J. Heinrich, "The role of cats and dogs in asthma and allergy - a systematic review," International Journal of Hygiene and Environmental Health, vol. 213, no. 1, pp. 1-31, 2010.

[12] B. J. Apelberg, Y. Aoki, and J. J. K. Jaakkola, "Systematic review: exposure to pets and risk of asthma and asthma-like symptoms," Journal of Allergy and Clinical Immunology, vol. 107, no. 3, pp. 455-460, 2001.

[13] N. Pearce, J. Douwes, and R. Beasley, "Is allergen exposure the major primary cause of asthma?" Thorax, vol. 55, no. 5, pp. 424-431, 2000.

[14] B. Takkouche, F. J. González-Barcala, M. Etminan, and M. Fitzgerald, "Exposure to furry pets and the risk of asthma and allergic rhinitis: a meta-analysis," Allergy, vol. 63, no. 7, pp. 857-864, 2008.

[15] S. L. Prescott, P. Smith, M. Tang et al., "The importance of early complementary feeding in the development of oral tolerance: concerns and controversies," Pediatric Allergy and Immunology, vol. 19, no. 5, pp. 375-380, 2008.

[16] P. G. Holt and P. D. Sly, "Non-atopic intrinsic asthma and the 'family tree' of chronic respiratory disease syndromes," Clinical and Experimental Allergy, vol. 39, no. 6, pp. 807-811, 2009.

[17] D. Martino and S. Prescott, "Epigenetics and prenatal influences on asthma and allergic airways disease," Chest, vol. 139, no. 3, pp. 640-647, 2011.

[18] C. V. Breton, H. M. Byun, M. Wenten, F. Pan, A. Yang, and F. D. Gilliland, "Prenatal tobacco smoke exposure affects global and gene-specific DNA methylation," American Journal of Respiratory and Critical Care Medicine, vol. 180, no. 5, pp. 462-467, 2009.

[19] B. Schaub, J. Liu, S. Höppler et al., "Maternal farm exposure modulates neonatal immune mechanisms through regulatory
T cells," Journal of Allergy and Clinical Immunology, vol. 123, no. 4, article e775, pp. 774-782, 2009.

[20] N. Aichbhaumik, E. M. Zoratti, R. Strickler et al., "Prenatal exposure to household pets influences fetal immunoglobulin e production," Clinical and Experimental Allergy, vol. 38, no. 11, pp. 1787-1794, 2008.

[21] H. J. Schönberger, E. Dompeling, J. A. Knottnerus, S. Kuiper, C. Van Weel, and C. P. Van Schayck, "Prenatal exposure to mite and pet allergens and total serum IgE at birth in highrisk children," Pediatric Allergy and Immunology, vol. 16, no. 1, pp. 27-31, 2005.

[22] H. Bisgaard, A. Simpson, C. N. A. Palmer et al., "Geneenvironment interaction in the onset of eczema in infancy: filaggrin loss-of-function mutations enhanced by neonatal cat exposure," PLoS Medicine, vol. 5, no. 6, article e131, 2008.

[23] J. E. Gern, C. L. Reardon, S. Hoffjan et al., "Effects of dog ownership and genotype on immune development and atopy in infancy," Journal of Allergy and Clinical Immunology, vol. 113, no. 2, pp. 307-314, 2004.

[24] J. D. Bufford, C. L. Reardon, Z. Li et al., "Effects of dog ownership in early childhood on immune development and atopic diseases," Clinical and Experimental Allergy, vol. 38, no. 10, pp. 1635-1643, 2008.

[25] H. Pohlabeln, S. Jacobs, and J. Böhmann, "Exposure to pets and the risk of allergic symptoms during the first 2 years of life," Journal of Investigational Allergology and Clinical Immunology, vol. 17, no. 5, pp. 302-308, 2007.

[26] M. Kerkhof, A. Wijga, H. A. Smit et al., "The effect of prenatal exposure on total IgE at birth and sensitization at twelve months and four years of age: the PIAMA study," Pediatric Allergy and Immunology, vol. 16, no. 1, pp. 10-18, 2005.

[27] P. Nafstad, P. Magnus, P. I. Gaarder, and J. J. K. Jaakkola, "Exposure to pets and atopy-related diseases in the first 4 years of life," Allergy, vol. 56, no. 4, pp. 307-312, 2001.

[28] S. T. Remes, J. A. Castro-Rodriguez, C. J. Holberg, F. D. Martinez, and A. L. Wright, "Dog exposure in infancy decreases the subsequent risk of frequent wheeze but not of atopy," Journal of Allergy and Clinical Immunology, vol. 108, no. 4, pp. 509-515, 2001.

[29] S. Sanderson, I. D. Tatt, and J. P. T. Higgins, "Tools for assessing quality and susceptibility to bias in observational studies in epidemiology: a systematic review and annotated bibliography," International Journal of Epidemiology, vol. 36, no. 3, pp. 666-676, 2007.

[30] P. P. Van Asperen and A. Mukhi, "Role of atopy in the natural history of wheeze and bronchial hyper-responsiveness in childhood," Pediatric Allergy and Immunology, vol. 5, no. 3, pp. 178-183, 1994.

[31] R. J. Bertelsen, K. C. L. Carlsen, B. Granum et al., "Do allergic families avoid keeping furry pets?” Indoor Air, vol. 20, no. 3, pp. 187-195, 2010.

[32] C. Svanes, J. P. Zock, J. Antó et al., "Do asthma and allergy influence subsequent pet keeping? An analysis of childhood and adulthood," Journal of Allergy and Clinical Immunology, vol. 118, no. 3, pp. 691-698, 2006.

[33] NHMRC, NHMRC Levels of Evidence and Grades for Recommendations for Developers of Guidelines, Council NHaMR, Canberra, Australia, 2009.

[34] S. Polk, J. Sunyer, L. Muñoz-Ortiz et al., "A prospective study of Fel $\mathrm{d} 1$ and Der p1 exposure in infancy and childhood wheezing," American Journal of Respiratory and Critical Care Medicine, vol. 170, no. 3, pp. 273-278, 2004.

[35] R. J. Kurukulaaratchy, S. Matthews, S. T. Holgate, and S. H. Arshad, "Predicting persistent disease among children who 
wheeze during early life," European Respiratory Journal, vol. 22, no. 5, pp. 767-771, 2003.

[36] L. A. Lowe, A. Simpson, A. Woodcock, J. Morris, C. S. Murray, and A. Custovic, "Wheeze phenotypes and lung function in preschool children," American Journal of Respiratory and Critical Care Medicine, vol. 171, no. 3, pp. 231-237, 2005.

[37] F. D. Martinez, A. L. Wright, L. M. Taussig et al., "Asthma and wheezing in the first six years of life," The New England Journal of Medicine, vol. 332, no. 3, pp. 133-138, 1995.

[38] S. Lau, S. Illi, T. A. E. Platts-Mills et al., "Longitudinal study on the relationship between cat allergen and endotoxin exposure, sensitization, cat-specific IgG and development of asthma in childhood-report of the German Multicentre Allergy Study (MAS 90)," Allergy, vol. 60, no. 6, pp. 766-773, 2005. 


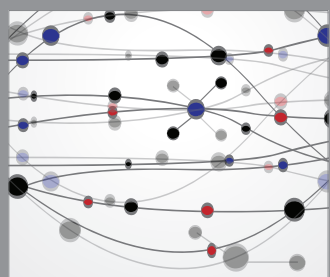

The Scientific World Journal
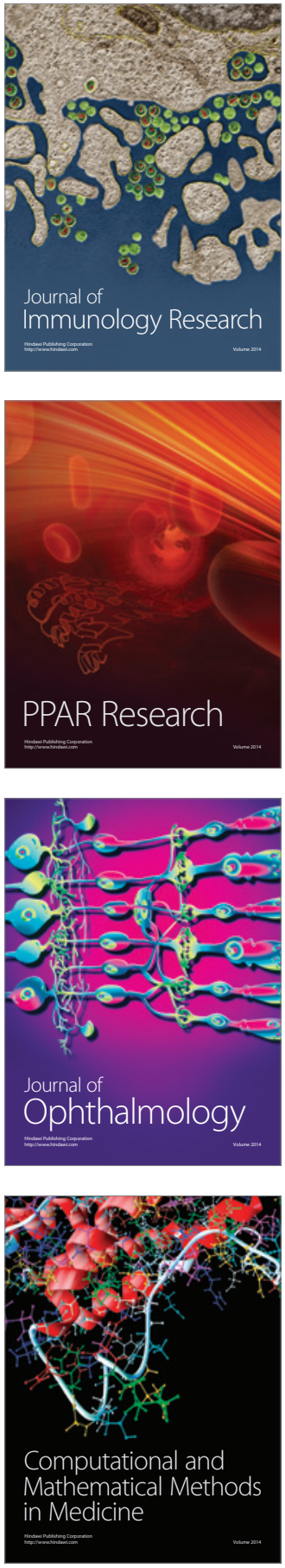

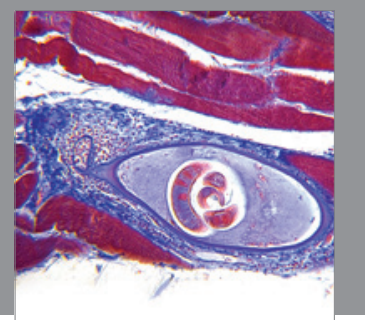

Gastroenterology

Research and Practice
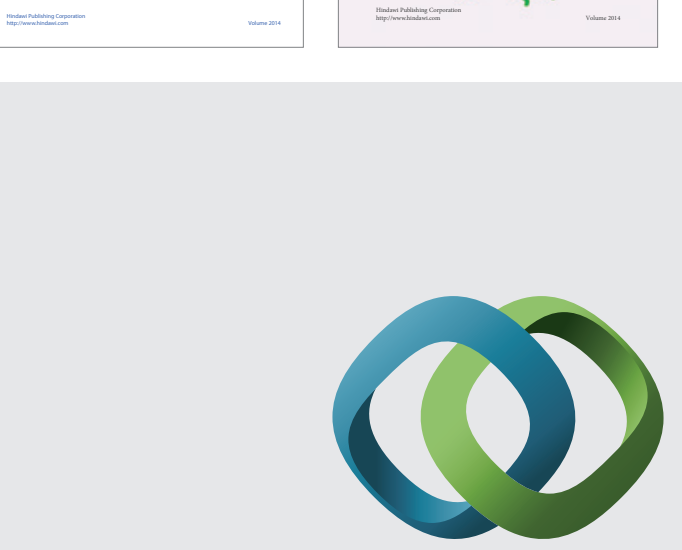

\section{Hindawi}

Submit your manuscripts at

http://www.hindawi.com
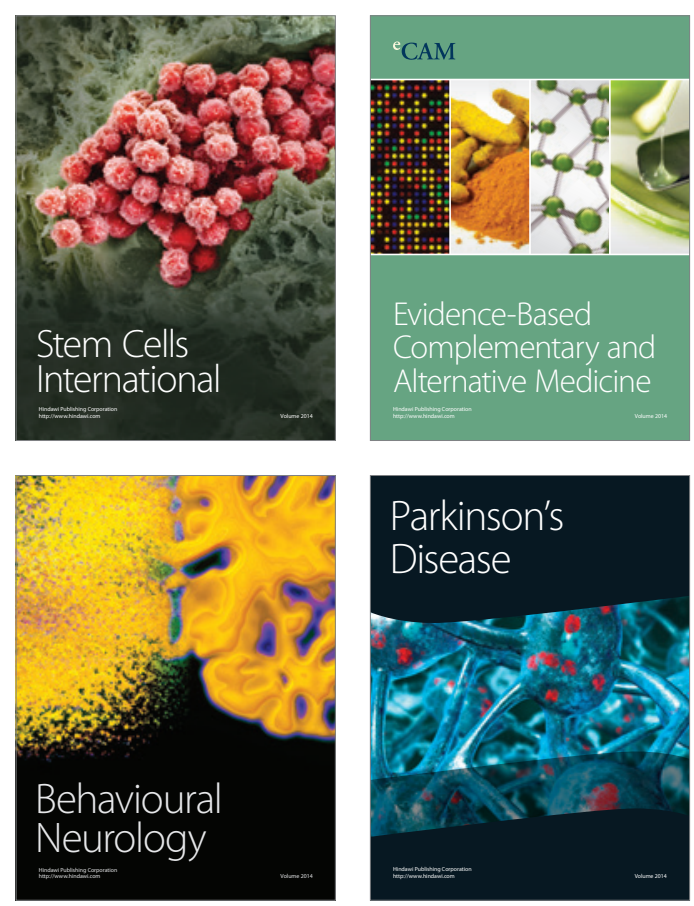

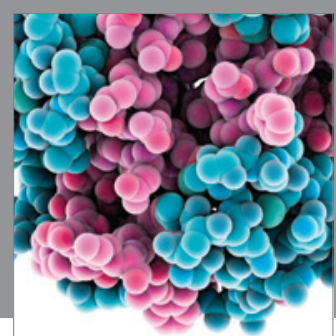

Journal of
Diabetes Research

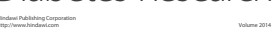

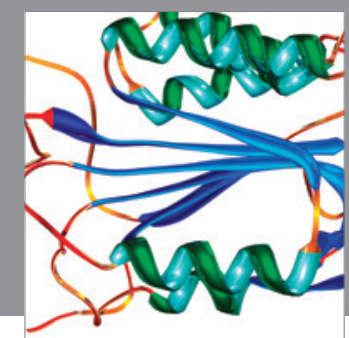

Disease Markers
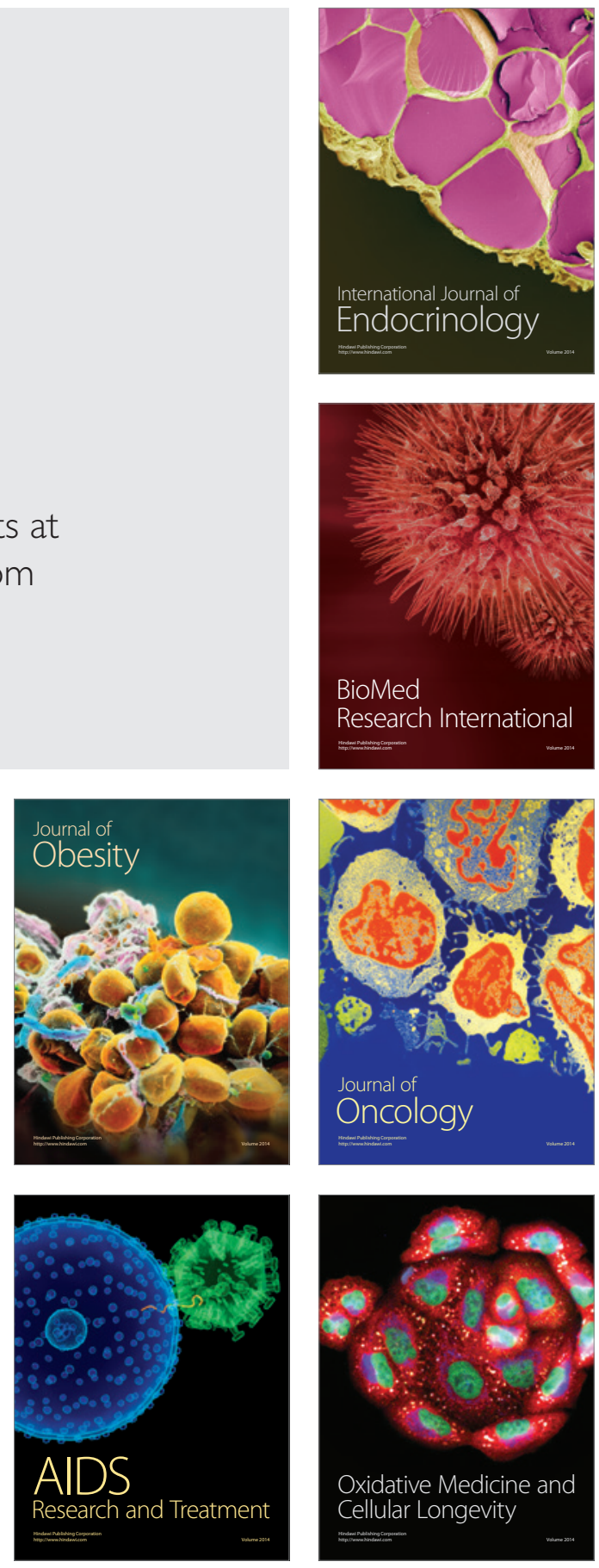